\title{
Screen printed frequency selective surfaces for room isolation in buildings
}

\author{
Aliya A. Dewani, Manimaran Kanesan, David V.Thiel, Steven G. O’Keefe and Mohammad Vatankhah \\ Varnoosfaderani \\ Centre for Wireless and Monitoring Applications (CWMA) \\ School of Engineering, Nathan Campus, Griffith University \\ 170, Kessels Road, Queensland, Australia \\ manimaran.kanesan@griffithuni.edu.au
}

\begin{abstract}
A ring type frequency selective surface (FSS) can provide transmission stop-band characteristics in rooms. This allows adjacent rooms to be isolated for one LAN for frequency reuse while other frequencies pass through the walls with minimal attenuation. The FSS was screen printed on a thin flexible plastic substrate of permittivity 3.2 with a stop band at $12.3 \mathrm{GHz}$ and $10 \mathrm{~dB}$ bandwidth of $3.5 \mathrm{GHz}$. The variation in bandstop characteristics was investigated for various wall materials. The centre frequency varied by more than $3 \mathrm{GHz}$ for common wall materials which means significant transparency for some building materials. The technique is a low cost method of confining LAN picocells in one room.
\end{abstract}

Keywords-printed FSS; ring FSS; FSS on target object; bandreject FSS, picocellular networks, transmission through walls;

\section{INTRODUCTION}

The number of wireless networks continues to increase with time. In buildings, picocellular communications, wireless sensor networks (WSN) and mobile telephone bands must coexist. While operating at very different frequencies, the challenge remains to ensure good connectivity for large area networks and well defined spatial limits for picocellular networks and WSN. There is also some interest in room isolation for data security. Frequency reuse and network security are two drivers to ensuring picocell isolation. A coating of metamaterial [1] is one option available to solve this problem, however the fabrication and installation costs must be very low to be economically viable [2]. As building materials include wood, paper (in plaster board), concrete and glass, a general purpose FSS which has similar isolation properties when placed against all of these materials is of interest.

The FSS is one type of metamaterial, a technique which employs a combination of dielectric materials and a regular array of sub-wavelength conductive objects. Such structures can be designed for band-reject transmission of microwave signals and is suitable for picocell isolation [3]. In the case of room isolation for a picocell, the bandstop frequency is most important. The ability to print conductors on a low cost, flexible plastic sheet means that installation on the walls and ceiling is relatively simple, and might even be incorporated into the wall materials themselves [4]. For example, prefabricated wall paneling could include the FSS structure without a significant increase in weight, volume or utility.
This paper discusses a printed FSS for picocell isolation inside a room. As the structure has no ground plane, the centre frequency of the stop band can be influenced by the electromagnetic properties of the wall materials the thickness of the wall. The effects on the FSS centre frequency was investigated for different target objects. The permittivity range of $1<\varepsilon_{r}<10$ and different thickness, $d$, are reported. While FSS characteristics have been reported previously, these studies have not commented on the FSS frequency changes with different wall materials.

The effect of various objects on the resonant frequency of printed RFID antennas has been reported [5], however, no simple calculation method exists to predict the change in resonant frequency. For this reason a full 3D electromagnetic modeling tool was required.

\section{FSS DESIGN}

The FSS used in this analysis is a conductive ring type. The rectangular array of rings was silver printed on top of substrate. This acts as a band stop filter. Figure 1 shows the dimension of the unit cell. The ring has width, $w=0.26 \mathrm{~mm}$ and radius, $r=$ $3.1 \mathrm{~mm}$ on flexible a thin plastic substrate of $\varepsilon_{r}=3.2$ with substrate thickness of $0.21 \mathrm{~mm}$. The structure was modeled in 3D electromagnetic software using a plane wave source excited normal to the plane of the conducting ring. The transmission properties were evaluated as a function of frequency of the incident radiation. Parker et al found that circular ring elements are less sensitive to incident angles when compared to other differently shaped elements [6]. On varying the angle of incidence from $0^{\circ}$ to $50^{\circ}$ the general features of the stop band stay the same as that for normal incidence. However for the TE mode transmission the higher order resonances start moving closer to resonant frequency whereas for TM mode transmission higher order resonances start moving away from the resonant frequency.

The transmission characteristics of the structure in air (i.e. setting the target object properties to those of air $\varepsilon_{r}=1$ and the conductivity $\sigma=0$ ), are plotted in Figure 2 as a function of frequency. The $10 \mathrm{~dB}$ bandwidth is $10.5 \mathrm{GHz}$ to $14.2 \mathrm{GHz}$ and is approximately symmetrical about the centre frequency at $12.35 \mathrm{GHz}$. This bandstop frequency changes with the substrate relative permittivity $\varepsilon_{r}$. Clearly if the properties of substrate and the object are both known, the structure can be optimized for a required frequency. In many cases, however, 
the electromagnetic properties of the object are not known, and can vary across the structure due to the addition of supporting beams and wall coatings.

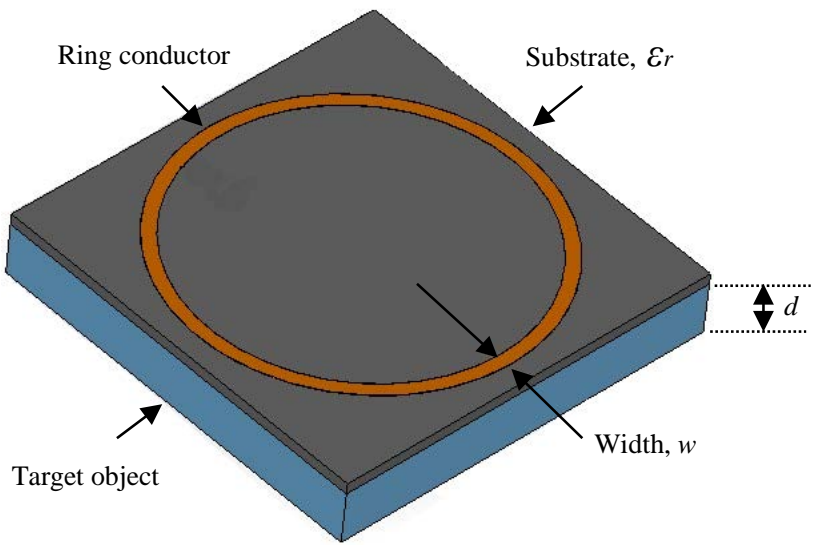

Fig. 1. Unit cell of the FSS structure consisting of a printed silver ring on top of a thin flexible plastic substrate and backed by target object.

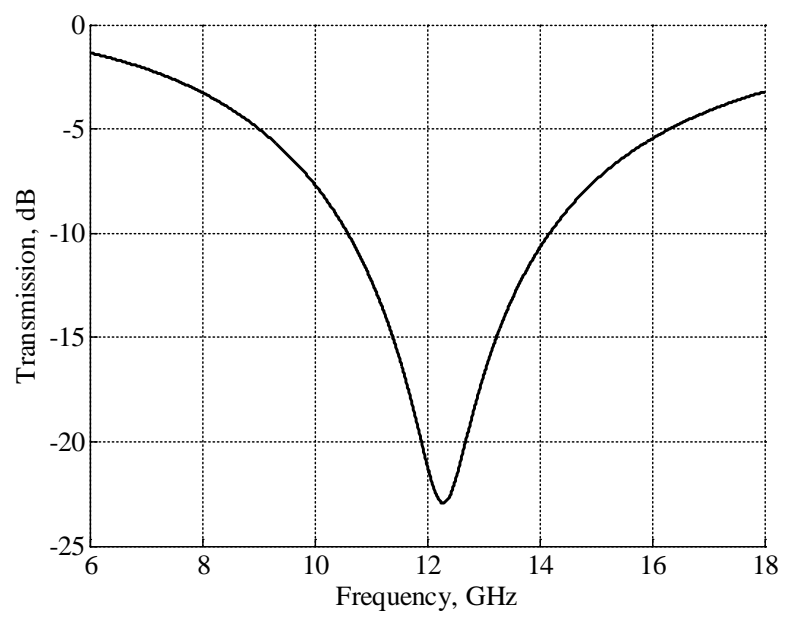

Fig. 2. FSS tranmission coefficient on a substrate with $\varepsilon_{r}=1$ and thickness $0.21 \mathrm{~mm}$. The $10 \mathrm{~dB}$ bandwidth is approximately $3.5 \mathrm{GHz}$.

\section{FSS ON DIFFERENT TARGET OBJECT}

When the FSS on a very thin substrate is placed on an object, the stopband frequency will change due to the different values of $\varepsilon_{r}$. The variation in the stopband frequency is shown in Figure 3 as a function of $\varepsilon_{r}$. The frequency decreases monotonically as the relative permittivity increases. For $\varepsilon_{r}>4$, the frequency shift is greater than the original bandwidth of the structure. This change is similar to the change in resonant frequency of a planar dipole antenna on a very thin substrate [7].

The analysis was extended to observe the effect a changing thickness $d$ of the target object. Figure 4 shows the variation in the stopband frequency with object thickness $d$. This range was designed to include most common materials used in wall, door and window construction. Thus the target object thickness $d$ was increased in $d$ from $1 \mathrm{~mm}$ to $20 \mathrm{~mm}$ for wood, concrete wall, glass and paper. The results shows there is no significant change in frequency shift when the $d$ changes. Note that the quantization in the frequency axis is the result of a computational accuracy in frequency of $0.1 \mathrm{GHz}$.

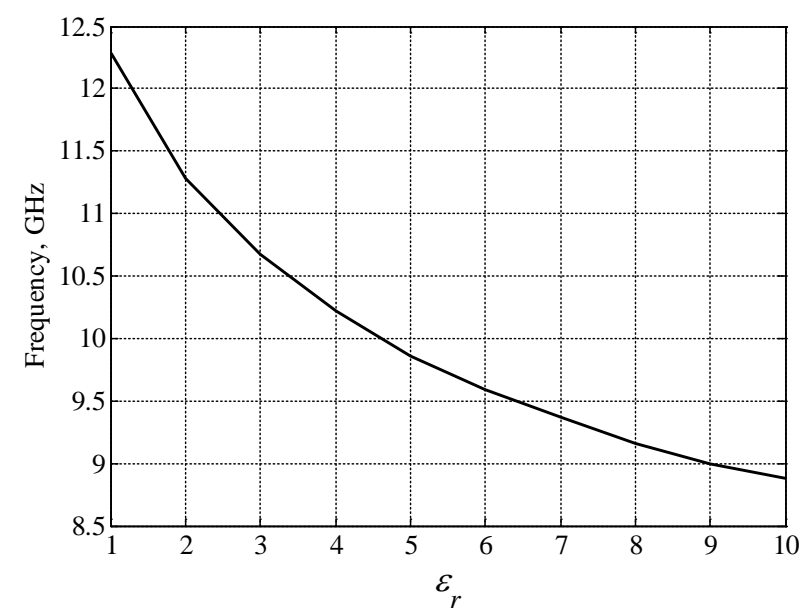

Fig. 3. Change in the bandstop frequency of a printed circular ring FSS on a thin plastic substrate (thickness $0.21 \mathrm{~mm}$ ) when the sheet is placed against a target object of thickness $2 \mathrm{~mm}$ with varying relative permittivity.

The very minor change in the FSS stopband frequency for $d$ $>1 \mathrm{~mm}$ demonstrates that the thickness of the object is not an important parameter in the attenuation of the signal. Clearly the effect of the thickness on the capacitance between the rings is minimal.

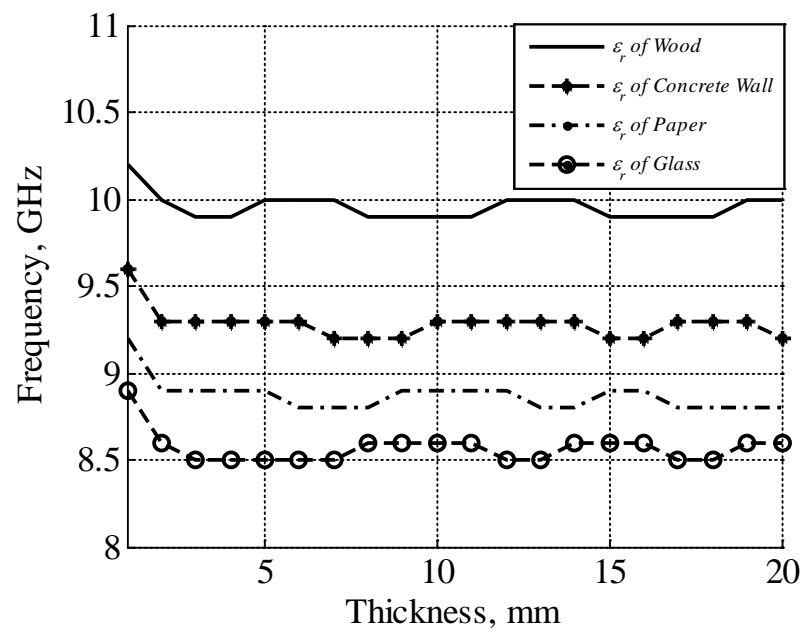

Fig. 4. FSS stopband frequency variations on different objects: wood $\left(\varepsilon_{r}=4\right)$, concrete $\left(\varepsilon_{r}=6\right)$, paper $\left(\varepsilon_{r}=8\right)$ and glass $\left(\varepsilon_{r}=10\right)[8,9]$ as a function of the thickness.

As the thickness of target object has no significant effect on the FSS stopband frequency, the effect is only dominated by the change in relative permittivity.

Figure 5 shows the transmission coefficient of the FSS on substrate with and without the target object. The FSS without target object is similar to Figure 2 and has a $3.5 \mathrm{GHz}$ 
bandwidth. With an object with $\varepsilon_{r}=10$ the bandwidth decreases to $2.5 \mathrm{GHz}$. Figure 5 clear demonstrates that the change is the centre frequency of the stopband is well outside the bandwidth of the FSS in air only. This is of significant importance in room isolation technologies. The printed FSS sheets designed for use on wood and concrete will not function satisfactorly when placed on glass.

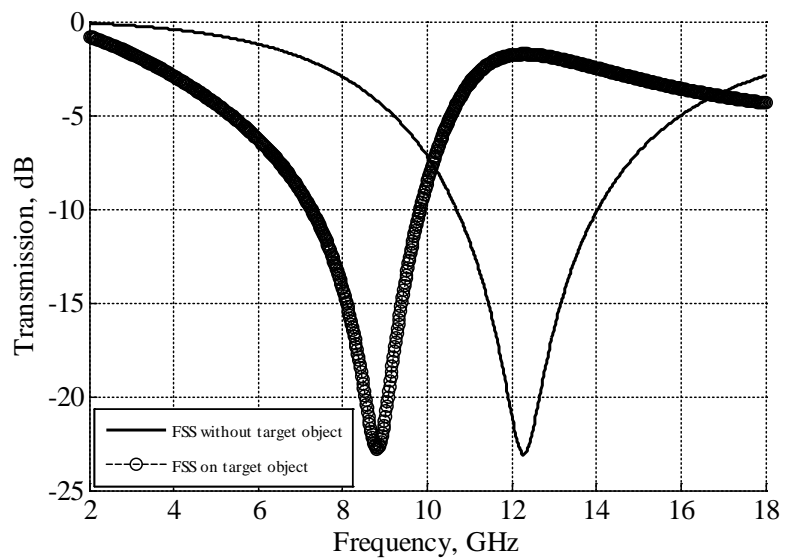

Fig. 5. FSS without target object (continuous line) and on $\varepsilon_{r}=10$ (o). The bandwidth is reduced by approximately $1 \mathrm{GHz}$. This plot demonstrates that this FSS can not be used effectively on these two different materials.

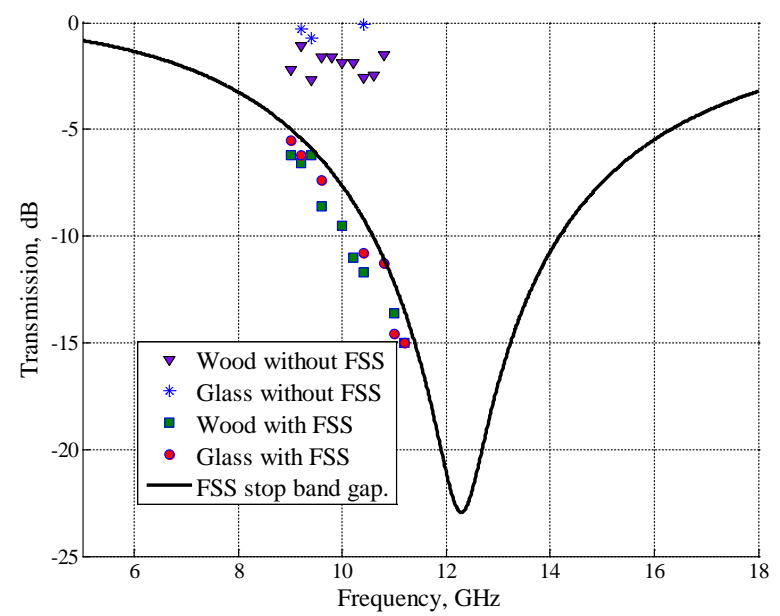

Fig. 6. S21 experimental results showing effect of the FSS on wood (thickness $=20 \mathrm{~mm}$ ) and glass (thickness $=10 \mathrm{~mm}$ ) compared to the wood and glass alone. The continuous line is the free space characteristic. The materials without the FSS coating show very little change with frequency.

The transmission coefficient (S21) was measured using two X-band pyramidal horn antennas placed $30 \mathrm{~cm}$ apart facing each other and $14.5 \mathrm{~cm}$ from the ground. The sample materials were placed halfway between the two horns and the response was measured as a function of frequency. The system was first calibrated using a free space path. The maximum generator frequency was $11.2 \mathrm{GHz}$ and the sensitivity of the receiver was $-35 \mathrm{dBm}$. Figure 6 shows that the attenuation (S21) characteristics when the thin plastic FSS was placed on wood and on glass. The measured free space path loss is the function of frequency. The S21 transmission characteristics for FSS on two different materials (wood and glass) were calculated by subtracting the free space variation (Wood ${ }_{\text {FsS }}$-Foam Plastic) and so is the glass with FSS (Glass FsS-Foam $_{\text {plastic). The wood }}$ without the FSS was found to be frequency independent and has a mean absorption loss of $-2.03 \mathrm{~dB}$. The loss through glass was also frequency independent and has a mean loss of $0.58 \mathrm{~dB}$. While the frequency range of the equipment was limited to below the stopband frequency, a statistical t-test was used to assess the difference between the two data sets. The results generated by this test are pooled variance, an accumulated measure of the spread of data about the mean, which is derived from this formula:

$$
S^{2}=\frac{n_{1} S_{1}^{2}+n_{2} S_{2}^{2}}{n_{1}+n_{2}-2}
$$

where $n_{1}$ and $n_{2}$ are the number of observations for wood with the FSS and glass with the FSS respectively. The $S_{1}$ and $S_{2}$ are the mean values for the wood and glass respectively. The results showed that there is $80 \%$ level of significance to support the fact that the mean test values of two target objects are different. However, it is clear that the stop band center frequency is greater than $11.2 \mathrm{GHz}$ for both wood and glass materials, but the shape of the curve is convincing that there is a clear stop band for both materials resulting from the FSS.

\section{CONCLUSION}

This paper demonstrates the impact of a low cost, FSS created by printing silver on a thin, flexible, plastic substrate and placed on various target objects used in the building industry. The relative permittivity of the target object can have a large and significant impact on the FSS bandstop centre frequency. The thickness of the target object showed little effect on the bandstop resonance. The bandwidth is reduced with increasing values of $\varepsilon_{r}$, particularly on high $\varepsilon_{r}$ objects. This work demonstrates that the printed FSS can provide good isolation in a room if the object is designed for and placed on one particular type of building material. When there are a number of different object materials, then the bandwidth might be too narrow to accommodate the large variations in the centre stopband frequency. This means that there will be significant microwave transmission through the windows (for example). There are two possible strategies to overcome this problem:

a) The FSS needs to be constructed in such a manner that the bandwidth is increased. This can be achieved using differently sized FSS conductive rings on the same substrate. This needs further investigation.

b) The FSS needs to be designed specifically for the objects. Thus a prefabricated wall will have one geometrical structure and the windows must have another. The wall FSS will have a different stopband characteristic in free space compared to the glass FSS. When these FSS coatings are applied to the different materials enclosing the room, the stopband frequency is the same. 
Note that the printed FSS has very good optical transparency so the windows remain quite transparent. The size and thickness of the conductive tracks is similar to the effect of insect screens commonly used in buildings.

\section{REFERENCES}

[1] B.A. Munk, Frequency selective surfaces: Theory and design, WileyInterscience, New York, 2000

[2] G.H.-H Sung, K.W. Sowerby, M.J. Neve, and A.G. Williamson, “A frequency-selective wall for interference reduction in wireless indoor environments," IEEE Antenna and Prop. Magazine, vol. 48, pp. 29-37, Oct 2006.

[3] R. Mittra, C.H. Chan and T. Cwik "Techniques for analyzing frequency selective surfaces-a review,” Proc. of the IEEE, vol. 76, pp. 1593-1615, Dec 1998.
[4] T.K. Wu, Frequency Selective Surface and Grid Array, John Wiley \& Sons, New York, 1995.

[5] M. Kanesan, D.V. Thiel and S.G. O'Keefe, "The effect of lossy dielectric objects on UHF RFID meander line antenna”. IEEE Antennas and Propagation Symposium, pp. 1-2, July 2012.

[6] E.A. Parker and S. M. A. Hamdy, "Rings as elements for frequency selective surfaces,” Electronics Letters, vol. 17 (17), pp. 612-614, Aug. 1981.

[7] M. Kanesan, D.V. Thiel, A.Galehdar and S.O'Keefe, "Rapid analysis and optimization of planar Yagi-Uda dipole arrays printed on a dielectric substrate,” Int J RF and Microwave CAE, DOI: 10.1002/mmce.20747, May 2013.

[8] M. Sadiku, Elements of Electromagnetics, 5th ed. Oxford University Press, Oxford, 2009.

[9] J.L. Davis and A.P. Anan , "Ground-penetrating radar for high-resolution mapping of soil and rock stratigraphy,” Geophysical Prospecting, vol.37, pp 531-551, April 2006. 